\title{
3D reverse-time migration using the acoustic wave equation: An experience with the SEG/EAGE data set
}

\author{
KWanguIn Yoon, University of Houston, Texas, U.S. \\ Changsoo SHin and Sangyong SuH, Seoul National University \\ LARRY R. LINES, University of Calgary, Alberta, Canada \\ SOONDUK HONG, KIGAM, Seoul, Korea
}

$\mathbf{K}_{\mathrm{i}}$

irchhoff is the most commonly used 3D prestack migration algorithm because of its speed and other economic advantages, but it uses a high-frequency ray approximation to the wave equation and, therefore, has difficulties in imaging complex geologic structures where multipathing occurs (e.g., beneath rugose horizons such as faulted salt domes where traveltime calculations become difficult).

In contrast to Kirchhoff migration, reverse-time migration computes numerical solutions to the complete wave equation and, therefore, is potentially more accurate. But this technique is not popular in the industry because it is computationally intensive and expensive. However, we felt some recent developments would allow 3D reverse-time migration to be done relatively inexpensively on PC-based distributed memory clusters.

In order to examine this hypothesis, we implemented reverse-time migration on a PC cluster by using higher-order accuracy finite difference algorithms (Wu et al., 1996), an excitation time approach (Chang and McMechan, 1994), and variable grids (Mufti et al., 1996) to reduce memory and CPU time. We then used the pseudo-spectral method-following Gazdag (1981), Kosloff and Baysal (1982), and Fornberg (1987) — to further reduce CPU time and core memory requirements.

In this article, we compare our reverse-time migration images with first-arrival Kirchhoff migration images to demonstrate that $3 \mathrm{D}$ reverse-time migration can produce high fidelity images under the PC-based distributed memory cluster machine.

Application to the SEG/EAGE salt model data. The SEG/EAGE salt model has a complicated structural nature, which is representative of salt intrusions in the Gulf of Mexico. It is a challenging standardized test for 3D prestack depth migration algorithms and thus well suited for our comparison of reverse-time migration and Kirchhoff migration (using first-arrival traveltimes). Figure 1 shows the velocity model for inline 490 or $x=9.78 \mathrm{~km}$. Figures 2 and 3 show the 3D reverse-time and Kirchhoff migrations, respectively, for this line. Figure 4 shows the velocity model for crossline 360 or y $=7.18 \mathrm{~km}$. Figures 5 and 6 show the 3D reverse-time and Kirchhoff migrations, respectively, for this line. For both the inline and crossline images, both reverse-time and Kirchhoff methods accurately image reflectors above the salt body. However, the subsalt Kirchhoff images are contaminated with noise and artifacts. In the reverse-time images, we can identify reflectors in their correct locations (although amplitudes are weak). The conclusion is that the base of salt image and the subsalt reflectors are defined better on the reverse-time migration than on the Kirchhoff migration.

Figure 7 shows the depth slice of the velocity model at 2.08 $\mathrm{km}$, which is just crossing the salt body. Figures 8 and 9 are depth slices of reverse-time and Kirchhoff migration images at the depth. Figure 10 is the horizontal slice through the subsalt region at $2.98 \mathrm{~km}$. Figures 11 and 12 show depth slices for reverse-time migration and Kirchhoff migration at this depth. By comparing Figure 8 (reverse-time migration) with Figure

9 (Kirchhoff migration), we see that reverse-time migration

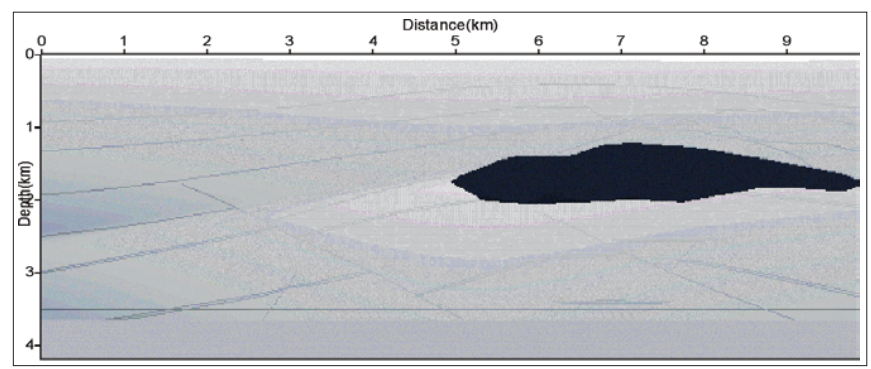

Figure 1. Section of 3D SEG/EAGE velocity model at inline 490, $x=9.78 \mathrm{~km}$.

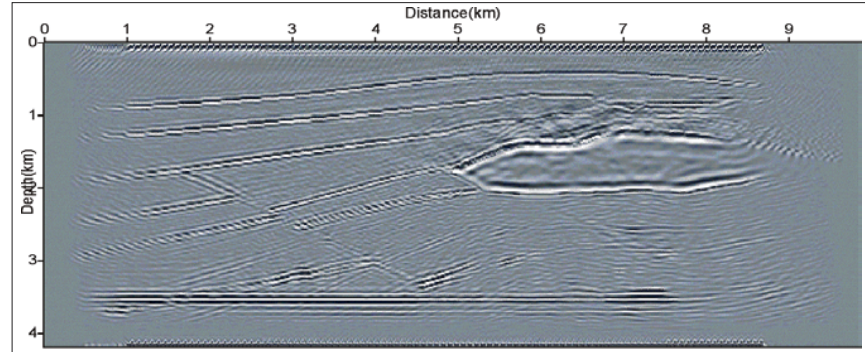

Figure 2. 3D reverse-time migration image of SEG/EAGE salt data at inline $490, x=9.78 \mathrm{~km}$.

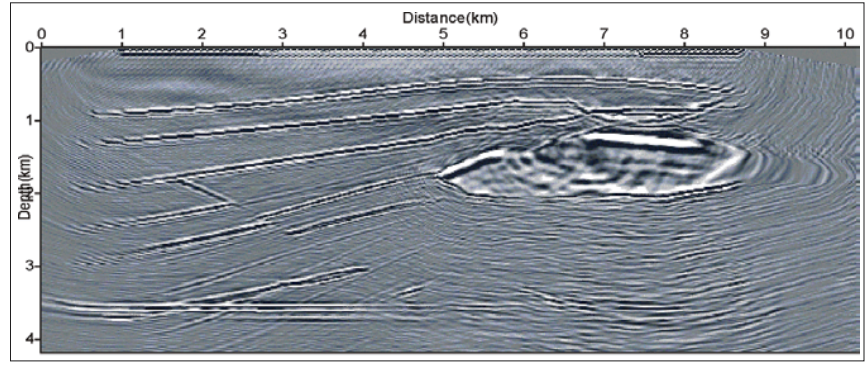

Figure 3. 3D first-arrival Kirchhoff migration image of SEG/EAGE salt data at inline 490, $x=9.78 \mathrm{~km}$.

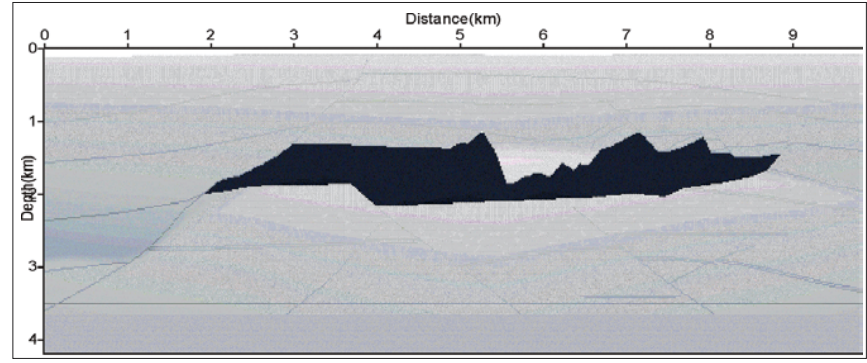

Figure 4. Section of 3D SEG/EAGE velocity model at crossline 360, $y=7.18 \mathrm{~km}$

gives a clearer, less contaminated view of the salt body. In Figure 11, we see weak fault lines and a clear salt boundary. However, the depth slice generated by Kirchhoff migration (Figure 12) does not clearly define the salt boundary or the subsalt reflectors. The reason for the improved images is that the use of the complete wave equation in reverse-time migration handles the large velocity contrasts better than the ray- 


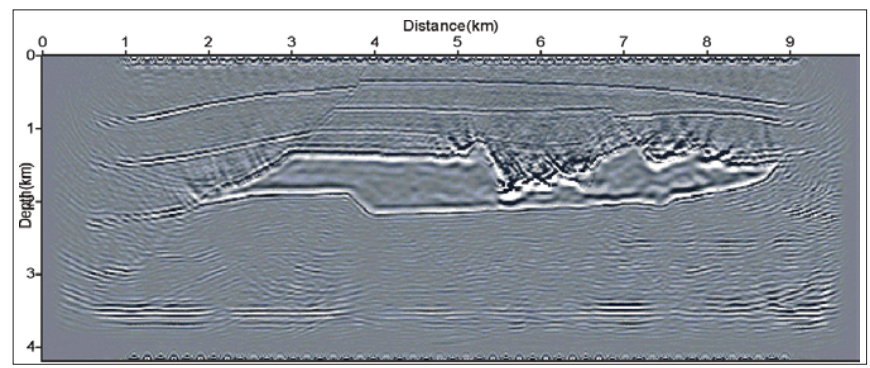

Figure $5.3 D$ reverse-time migration image of SEG/EAGE salt data at crossline $360, y=7.18 \mathrm{~km}$.

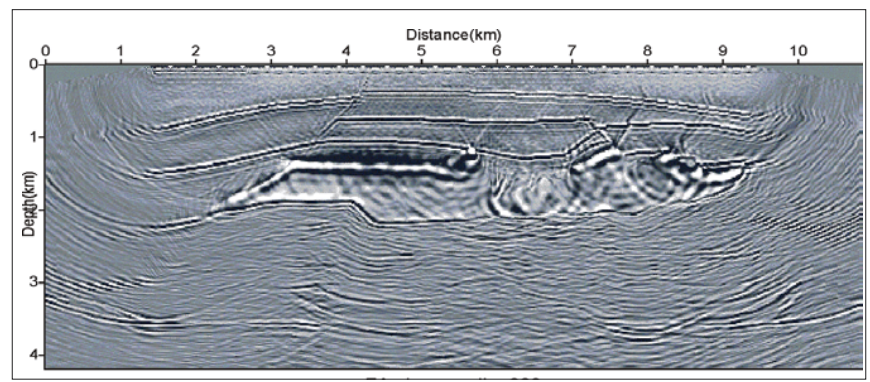

Figure 6. 3D first-arrival Kirchhoff migration image of SEG/EAGE salt data at crossline $360, y=7.18 \mathrm{~km}$.

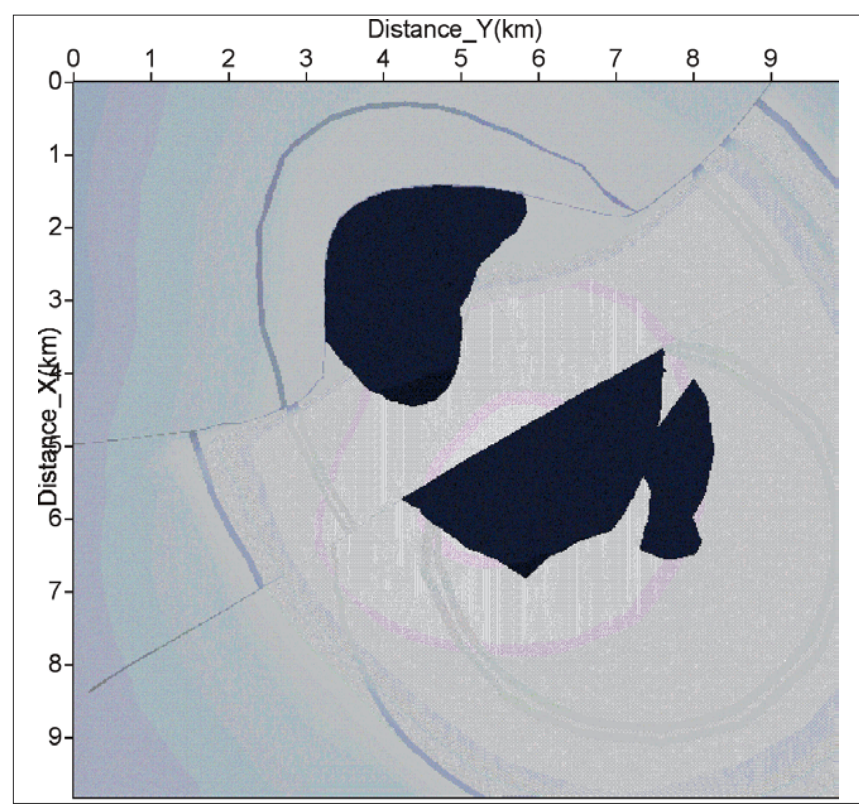

Figure 7. Depth-slice of 3D SEG/EAGE velocity model at depth grid 105, $z=2.08 \mathrm{~km}$, which goes through the salt body.

tracing approximations of Kirchhoff migration.

Computational resources. $3 \mathrm{D}$ prestack depth migration is the most computationally demanding processing step in exploration seismology. It is generally agreed that the most tractable solution to this problem resides in the use of parallel computing. Gavrilov et al. (2000) discussed how parallel computing algorithms are to be utilized by Kirchhoff and reverse-time migration algorithms. Kirchhoff is described as "embarrassingly parallel" because the depth images from a set of processors can be summed together to produce an image. For the reverse-time migration problem, Gavrilov et al. assigned each processor a part of the finite-difference calculation for a 3D grid. The processors communicate through a message passing interface (MPI). Gavrilov et al. showed increases of speed which were two orders of magnitude faster than with scalar processors.

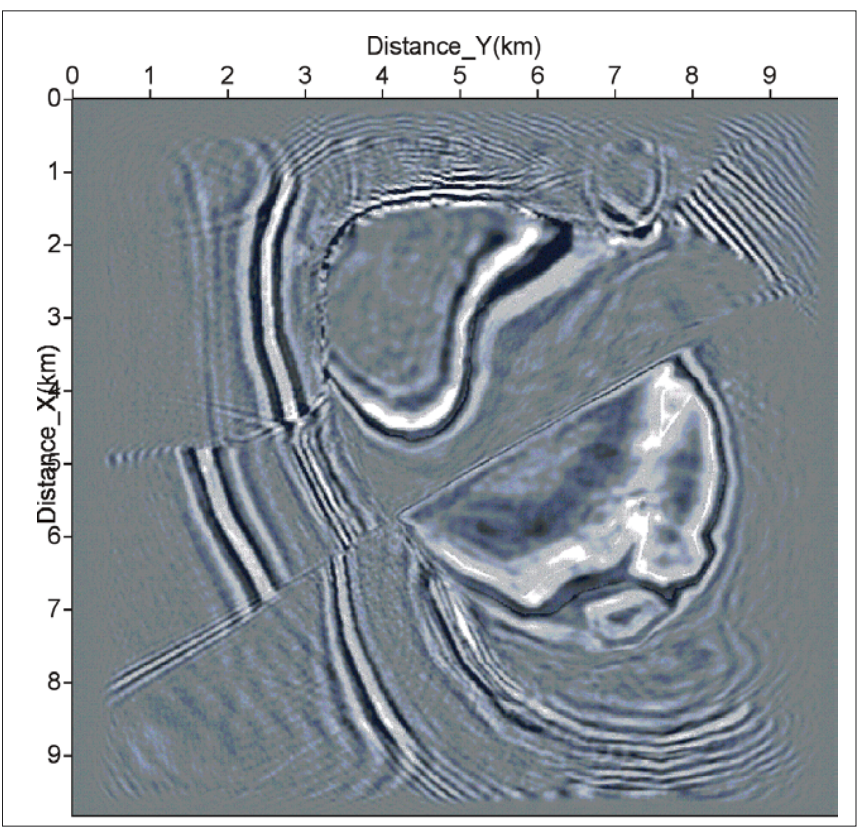

Figure $8.3 D$ reverse-time migration image of SEG/EAGE salt data at depth grid 105, $z=2.08 \mathrm{~km}$.

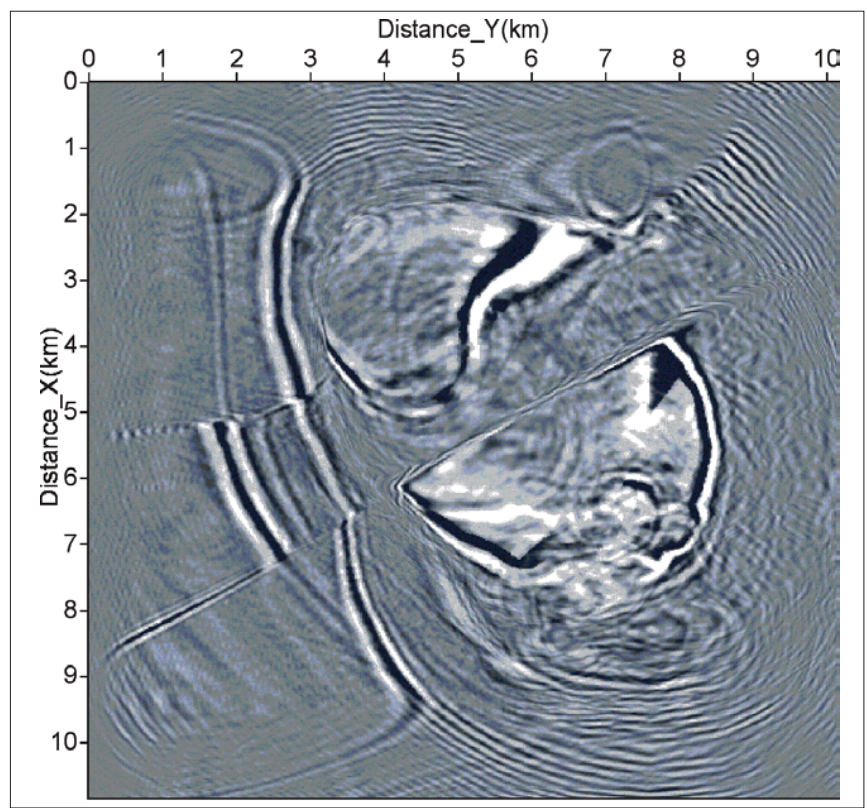

Figure 9. 3D first-arrival Kirchhoff migration image of SEG/EAGE salt data at depth grid 105, $z=2.08 \mathrm{~km}$.

In the computation of Green's functions, we did not apply the excitation time condition (Chang and McMechan, 1994) but the full wave equation modeling which included all wave events. We used a large image aperture- $-3.2 \mathrm{~km}$ crossline, 4.2 $\mathrm{km}$ inline, and $4.2 \mathrm{~km}$ depth which correspond to 160, 210, and 210 grid points of the SEG/EAGE salt dome model. Reverse-time migration of a shot-gathered data set took an average of seven hours of CPU time using an IBM Nighthawk Power3-II $375 \mathrm{MHz}$ CPU requiring $400 \mathrm{MB}$ of memory and 10 GB of disk space. For the 4800 shots, reverse-time migration took one month using 48 CPUs. For the Kirchhoff migration, we used a cluster of 32 Pentium IV CPUs. Kirchhoff migration required about two days using 26 CPUs and a dynamic image aperture whose radius varied from 0 to 1.5 $\mathrm{km}$. Our implementation of reverse-time migration was therefore about 15 times as expensive as our Kirchhoff migration. The expense of reverse-time migration depends on grid 


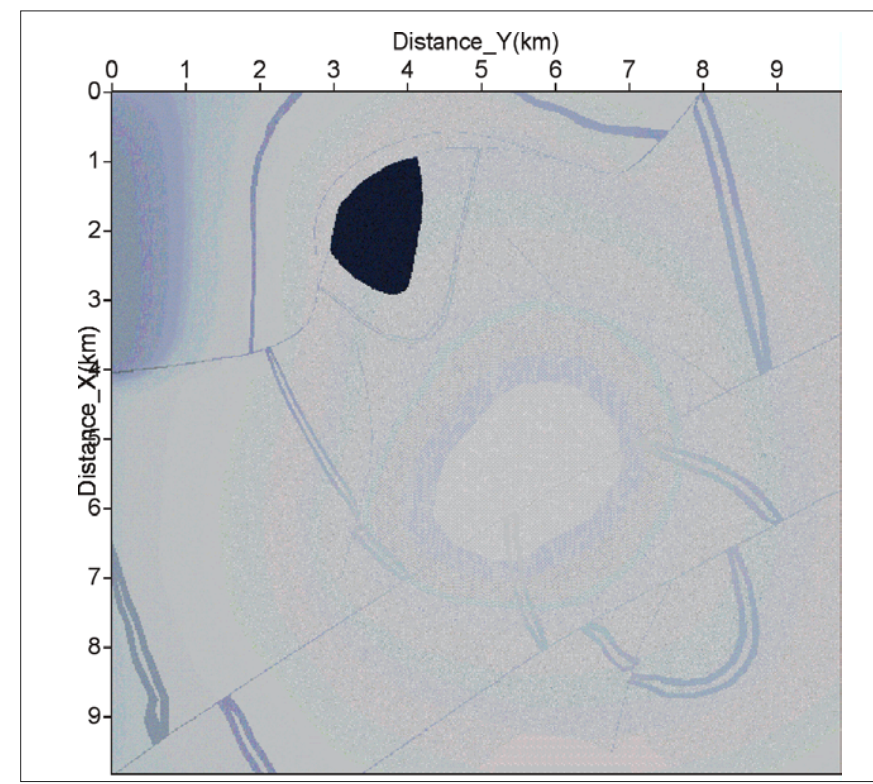

Figure 10. Depth-slice of 3D SEG/EAGE velocity model at depth grid $150, z=2.98 \mathrm{~km}$, which crosses the subsalt region.

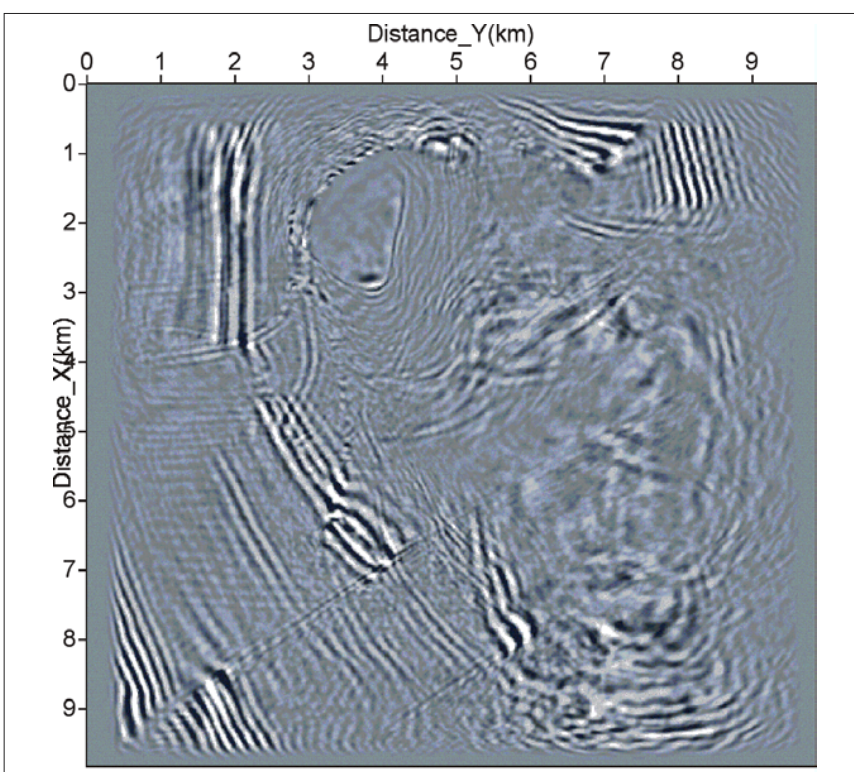

Figure 11. 3D reverse-time migration image of SEG/EAGE salt data at depth grid $150, z=2.98 \mathrm{~km}$.

size. The grid size depends on the wavelength of the dominant seismic energy which, in turn, depends on the layer velocities and the dominant seismic frequencies. The SEG/EAGE salt synthetic data has a major frequency bandwidth of $15-30 \mathrm{~Hz}$. With the grid size of $20 \mathrm{~m}$ used in this application, we can propagate acoustic wavefields within the frequency bandwidth of $0-37.5 \mathrm{~Hz}$ using the pseudo-spectral method. If we wanted to image data that has $75 \mathrm{~Hz}$ as a maximum frequency, the grid size should be reduced to $10 \mathrm{~m}$. This reduced grid size would increase computational cost and memory for a 3D problem by eight times. Each node of the current distributed memory cluster machine would not have enough memory to apply this reverse-time migration technique to real data without use of the message passing interface (MPI) programming. However, we can easily overcome such a difficulty using current shared memory processor (SMP) machines. Moreover, following Mufti et al. (1996), if we use variable grids, such as a hexahedron of $4.8 \times 6.4 \times 4.2$ $\mathrm{km}$, we can implement reverse-time migration using $75 \mathrm{~Hz}$ as fmax with 800 megabytes of core memory. This is an afford-

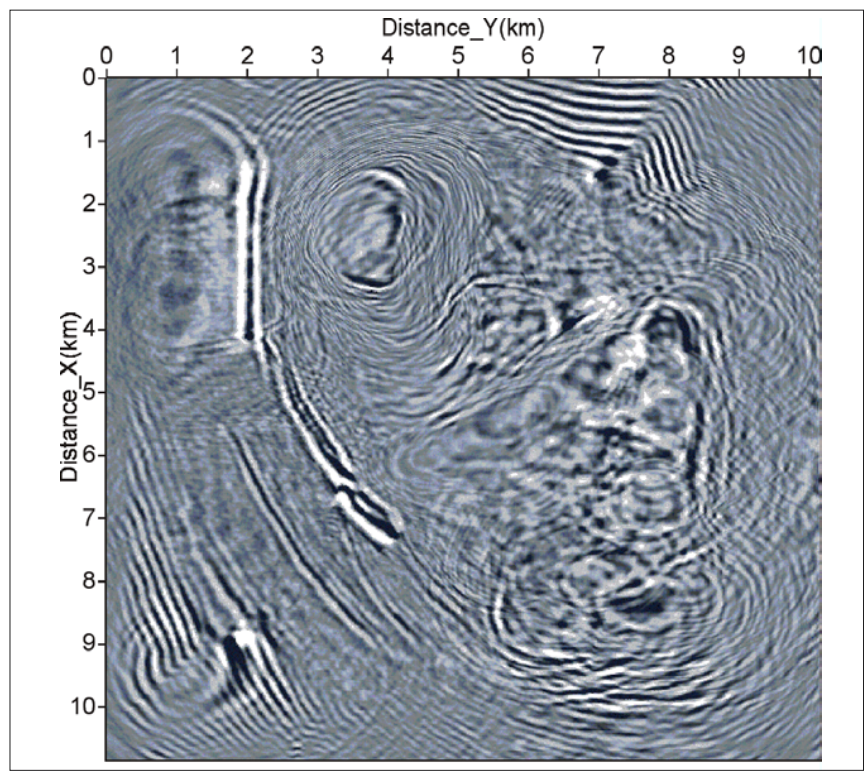

Figure 12. 3D first-arrival Kirchhoff migration image of SEG/EAGE salt data at depth grid 150, $z=2.98 \mathrm{~km}$.

able size. This parallel computing is easy to code and most efficient for prestack migrations.

Conclusions. Although many have attempted to image beneath the SEG/EAGE salt dome, the results have often been disappointing. To our knowledge, the results we have obtained by a brute 3D reverse-time migration based on the acoustic wave equation have provided some of the most accurate results to date. Even though 3D reverse-time migration is still very expensive compared with Kirchhoff migration, it is anticipated that we will be able, in the near future, to implement $3 \mathrm{D}$ reverse-time migration with ease using economical PCbased cluster machines.

Suggested reading. "Reverse-time migration" by Baysal et al. (GEOPHYSICS, 1983). "3D elastic prestack reverse-time migration" by Chang and McMechan (GEOPHYSICS, 1994). "The pseudospectral method: Comparisons with finite-differences for the elastic wave equation" by Fornberg (GEOPHYSICS, 1987). "3D depth migration: Parallel processing and migration movies" by Gavrilov et al. (TLE, 2000). "Modeling of the acoustic wave equation with transform methods" by Gazdag (GeOPHYSICS, 1981). "Equations d'onde et modeles" by Hemon (Geophysical Prospecting, 1978). "Forward modeling by a Fourier method" by Kosloff and Baysal (GEOPHYSICS, 1982). "Reversed time migration in spatial frequency domain" by Loewenthal and Mufti (GEOPHYSICS, 1983). "Migration by extrapolation of time-dependent boundary values" by McMechan (Geophysical Prospecting, 1983). "Finite-difference depth migration of exploration-scale 3-D seismic data" by Mufti et al. (GEOPHYSICS, 1996). "Iterative depth migration by backward time propagation" by Whitmore (SEG 1983 Expanded Abstracts). "Twodimensional post-stack depth migration: A survey of methods" by Whitmore et al. (First Break, 1988). "Analysis of higher-order, finite-difference schemes in 3D reverse-time migration" by Wu et al. (GEOPHYSICS, 1996). "Comparison of Kirchhoff and reversetime migration methods with applications to prestack depth imaging of complex structures" by Zhu and Lines (GEOPHYSICS, 1998). $T_{L} E$

Acknowledgments: The authors thank the financial supporters of the Brain Korea 21 Project and the National Research Laboratory project of the Ministry of Science and Technology.

Corresponding author: lines@geo.ucalgary.ca 